\title{
Some aspects of vector-valued singular integrals
}

Oscar Blasco

Department of Mathematics, Universitat de Valencia, Burjassot 46100 (Valencia) Spain oblasco@uv.es ${ }^{\dagger}$

\section{Introduction and notation}

Two of the basic operators in Harmonic Analysis are the well-known "Hilbert transform" and "Hardy-Littlewood maximal operator". The Hardy-Littlewood maximal function is defined in $\mathbb{R}^{n}$ by

$$
M f(x)=\sup _{x \in Q} \frac{1}{|Q|} \int|f(y)| d y,
$$

where $Q$ is a cube in $\mathbb{R}^{n}$ and $|Q|$ stands for the Lebesgue measure of the cube.

The Hilbert transform is defined in $\mathbb{R}$ by several equivalent ways:

$$
\begin{gathered}
H f(x)=\frac{1}{\pi} \lim _{\varepsilon \rightarrow 0} \int_{|y|>\varepsilon} \frac{f(x-y)}{y} d y, \\
H f(x)=\lim _{t \rightarrow 0} Q_{t} * f(x),
\end{gathered}
$$

where $Q_{t}$ is the conjugate Poisson kernel, $Q_{t}(x)=\frac{1}{\pi} \frac{x}{x^{2}+t^{2}}$ or

$$
(H f)(\xi)=-i \operatorname{sgn}(\xi) \hat{f}(\xi) .
$$

Their boundedness properties in the Lebesgue spaces are rather wellknown and its possible generalizations to higher dimension, other spaces or its vector-valued analogues have been a source of creativity for mathematicians over the decades.

These operators are known to be of weak-type $(1,1)$,

$$
|\{x:|H f(x)|>\lambda\}| \leq C \frac{\|f\|_{1}}{\lambda}
$$

\footnotetext{
${ }^{\dagger}$ Partially supported by Proyecto MTM 2005-08350
} 


$$
|\{x:|M f(x)|>\lambda\}| \leq C \frac{\|f\|_{1}}{\lambda}
$$

and of strong-type $(p, p)$

$$
\begin{aligned}
& \|H f\|_{p} \leq C\|f\|_{p}, \quad 1<p<\infty, \\
& \|M f\|_{p} \leq C\|f\|_{p}, \quad 1<p \leq \infty .
\end{aligned}
$$

The endpoint results for $p=1$ and $p=\infty$ for the Hilbert transform lead to two very important spaces in Harmonic Analysis, namely $B M O(\mathbb{R})$ and $H^{1}(\mathbb{R})$.

$B M O(\mathbb{R})$ consists of locally integrable functions such that $\sup _{Q} \operatorname{osc}_{p}(f, Q)<$ $\infty$ for some (or equivalently for all) $0<p<\infty$, where

$$
\operatorname{osc}_{p}(f, Q)=\left(\frac{1}{|Q|} \int_{Q}\left|f(x)-f_{Q}\right|^{p} d x\right)^{1 / p}
$$

and $f_{Q}=\frac{1}{|Q|} \int_{Q} f(x) d x$ for a cube $Q$ in $\mathbb{R}$.

The space can also be described by means of the sharp maximal function of $f([\mathrm{FS}])$,

$$
f^{\#}(x)=\sup _{x \in Q} \frac{1}{|Q|} \int_{Q}\left|f(x)-f_{Q}\right| d x .
$$

Of course, $f \in B M O(\mathbb{R})$ if and only if $f^{\#} \in L^{\infty}(\mathbb{R})$ and the "norm" in $B M O$ is then given by $\left\|f^{\#}\right\|_{\infty}$.

Let us also recall that $B M O(\mathbb{R})$ is the dual space of $H^{1}(\mathbb{R})([\mathrm{FS}, \mathrm{C}])$ where $H^{1}$ is the Hardy space defined in terms of atoms, i.e. that is the space of integrable functions $f=\sum_{k} \lambda_{k} a_{k}, \lambda_{k} \in \mathbb{R}, \sum_{k}\left|\lambda_{k}\right|<\infty$ and where $a_{k}$ belong to $L^{\infty}(\mathbb{R}), \operatorname{supp}\left(a_{k}\right) \subset Q_{k}$ for some cube $Q_{k}, \int_{Q_{k}} a(x) d x=0$ and $|a(x)| \leq \frac{1}{\left|Q_{k}\right|}$. The norm is now given by the infimum of $\sum_{k}\left|\lambda_{k}\right|$ over all possible decompositions of $f$.

With these definitions out of the way, we can now mention that the Hilbert transform maps $H^{1}(\mathbb{R})$ into $L^{1}(\mathbb{R})$ and $L^{\infty}(\mathbb{R})$ into $B M O(\mathbb{R})$, i.e.

$$
\begin{gathered}
\|H f\|_{B M O} \leq C\|f\|_{\infty}, \\
\|H f\|_{1} \leq C\|f\|_{H^{1}} .
\end{gathered}
$$

There is another rather relevant connection between $B M O$ and the commutator between multiplication operators and singular integrals. It was first shown by Coifman, Rochberg and Weiss $([\mathrm{CRW}])$ that,denoting $H_{b}(f)=$ $b H(f)-H(b f)$, one has

$$
b \in B M O(\mathbb{R}) \text { if and only if } H_{b}: L^{2}(\mathbb{R}) \rightarrow L^{2}(\mathbb{R}) \text { is bounded } .
$$

There have been several directions in which results from (1) to (7) have been extended and developed. One approach, which goes back to the early 
fifties, was the consideration of convolution operators with kernels similar in higher dimensions. The big contribution to the general theory of operators that share similar properties with the Hilbert transform is due to the work of A. Calderón and A. Zygmund (see [CZ]).

I took some time for people to isolate the properties that were needed in the kernel in order to apply similar techniques to other classes of operators.

After some preliminary stages (see $[\mathrm{S}],[\mathrm{H}]$ ) in the case of convolution kernels, the theory was then extended to non-convolution operators (see $[\mathrm{CM}]$ or $[J])$.

Following Coifman and Meyer we shall say that $T$ is a generalized CalderónZygmund operator if it has following properties:

(i) $T$ is bounded on $L^{2}\left(\mathbb{R}^{n}\right)$,

(ii) there exists a locally integrable function $k$ from $\mathbb{R}^{n} \times \mathbb{R}^{n} \backslash\{(x, x): x \in$ $\left.\mathbb{R}^{n}\right\}$ into $\mathbb{C}$ such that

$$
T f(x)=\int k(x, y) f(y) d y
$$

for every bounded and compactly supported function $f$ and $x \notin$ supp $f$,

(iii) there exists $\varepsilon>0$ such that the kernel satisfies

$$
\begin{gathered}
|k(x, y)| \leq \frac{C}{|x-y|}, \\
\left|k(x, y)-k\left(x^{\prime}, y\right)\right| \leq C \frac{\left|x-x^{\prime}\right|^{\varepsilon}}{|x-y|^{n+\varepsilon}},|x-y| \geq 2\left|x-x^{\prime}\right|, \\
\left|k(x, y)-k\left(x, y^{\prime}\right)\right| \leq C \frac{\left|y-y^{\prime}\right|^{\varepsilon}}{|x-y|^{n+\varepsilon}},|x-y| \geq 2\left|y-y^{\prime}\right| .
\end{gathered}
$$

In general, for generalized Calderón-Zygmund operators one does not have the identity

$$
T f(x)=\lim _{\varepsilon \rightarrow 0} \int_{|x-y|>\varepsilon} k(x, y) f(y) d y, \quad f \in \mathcal{S}\left(\mathbb{R}^{n}\right) .
$$

The operators where (12) happens to hold true were called Calderón-Zygmung type singular integrals.

For such operators one defines the corresponding maximal operator

$$
T^{*}(f)(x)=\sup _{\varepsilon>0}\left|\int_{|x-y|>\varepsilon} K(x, y) f(y) d y\right| .
$$

Let us collect in the following theorem the boundedness properties of such operators.

Theorem 1.1 Let $T$ be a Calderón-Zygmund type singular operator defined on $\mathbb{R}^{n}$. Then

$$
|\{x:|T f(x)|>\lambda\}| \leq C \frac{\|f\|_{1}}{\lambda},
$$




$$
\begin{gathered}
\|T f\|_{1} \leq C\|f\|_{H^{1}}, \\
\|T f\|_{p} \leq C\|f\|_{p}, \quad 1<p<\infty, \\
\|T f\|_{B M O} \leq C\|f\|_{\infty}, \\
\left|\left\{x:\left|T^{*} f(x)\right|>\lambda\right\}\right| \leq C \frac{\|f\|_{1}}{\lambda}, \\
\left\|T^{*} f\right\|_{p} \leq C\|f\|_{p} \quad 1<p<\infty .
\end{gathered}
$$

It is known (see $[\mathrm{D}]$ Theorem 5.12) that to get the boundedness and the weak-type $(1,1)$ for such operator conditions $(9),(10)$ and (11) can be replaced by the Hörmander type ones

$$
\begin{aligned}
& \int_{|x-y| \geq 2\left|x-x^{\prime}\right|}\left|k(x, y)-k\left(x^{\prime}, y\right)\right| \leq C, \\
& \int_{|x-y| \geq 2\left|y-y^{\prime}\right|}\left|k(x, y)-k\left(x, y^{\prime}\right)\right| \leq C .
\end{aligned}
$$

Another direction, which we shall consider throughout this paper, is the vector-valued consideration of the problem.

There are two different points of view. One is to consider sequences of functions $\left(f_{j}\right)$, rather than for a single one, and ask ourselves whether inequalities like

$$
\left\|\left(\sum_{j}\left|H f_{j}\right|^{r}\right)^{1 / r}\right\|_{p} \leq C\left\|\left(\sum_{j}\left|f_{j}\right|^{r}\right)^{1 / r}\right\|_{p}
$$

hold true for some values $0<p, r<\infty$.

Another one is to analyze whether inequalities like

$$
\left\|\left(\sum_{j}\left|T_{j} f\right|^{r}\right)^{1 / r}\right\|_{p} \leq C\|f\|_{p}
$$

hold true for some values $0<p, r<\infty$, where $T_{j}$ are Calderón-Zygmund operators with some assumptions.

These results, nowadays, fit in a theory of general vector-valued functions, when allowing either the function or the kernel to take values in Banach spaces.

A systematic study and its applications to Littlewood-Paley theory or maximal functions was first started in the work of Benedek, Calderón and Panzone ([BCP]) and then continued by Rubio de Francia, Ruiz and Torrea ([RRT].

The vector-valued theory also has some applications in a more abstract setting and interplays with the geometry of Banach spaces when analyzing the properties on the Banach space for several classical results to hold true in the Banach-valued setting.

One of the most important vector-valued extension is the following: Let $X$ be a Banach space and consider $f=\sum_{k=1}^{m} \phi_{k} x_{k}$ where $\phi_{k} \in L^{p}(\mathbb{R})$ and $x_{k} \in X$. We can now define the vector-valued Hilbert transform 


$$
H f(x)=\frac{1}{\pi} \lim _{\varepsilon \rightarrow 0} \int_{|y|>\varepsilon} \frac{f(x-y)}{y} d y=\sum_{k=1}^{m} H\left(\phi_{k}\right) x_{k} \in X .
$$

The main question is to know whether the operator, defined on $L^{p}(\mathbb{R}) \otimes X$, extends continuously to $L_{X}^{p}(\mathbb{R})$. A positive answer for $X=\ell^{r}$ answers the question in (15). In this direction it is not difficult to see that for $X=\ell^{1}(\mathbb{N})$ the operator $H \otimes I d$ is not bounded on $L_{\ell^{1}}^{2}(\mathbb{R})$. However it follows from a general $\ell^{2}$-valued extension result due to Marcinkiewicz and Zygmund (see [GR],Theorem 2.7) that (15) holds for the case $X=\ell^{2}(\mathbb{N})$. The description of the property on the space $X$ for the boundedness of the vector-valued Hilbert transform goes back to the work of Burkholder and Bourgain (see [Bu1, Bo1] ) who proved to be equivalent to the boundedness of the unconditional martingale differences transform and, since then on called the UMD property.

From this point of view, and realizing that the boundedness on $L^{2}$ is equivalent to the one in any $L^{p}$ for $1<p<\infty$, one can say that (15) holds for $1<p, r<\infty$ because $\ell_{r}$ has the UMD property in the case $1<r<\infty$.

The UMD property became rather relevant, due to the interplay of the Hilbert transform with orther areas whenever functions were allowed to take values in Banach spaces.

Another example of the use of vector-valued analysis in connection with classical operators comes from the Banach-lattice-valued extension of the Hardy-Littlewood maximal function ([GMT]). A Banach function space $L$ is said to have the Hardy-Littlewood property if

$$
M_{L}(f)(x)=\sup _{x \in Q} \frac{1}{|Q|} \int_{Q}|f(x)| d x,
$$

where the supremum is taken in the lattice structure. Several characterization and results concerning this property were achieved in [GMT, GMT2].

I would like to point out here that Carlos Segovia got interested in the interplay between the geometry of Banach spaces and the vector-valued analysis and we refer the reader to his work in [HMST] for a combination of techniques from $A_{p}$ theory, Harmonic Analysis and the use of Hardy-Littlewood property in Banach lattices.

As for the Hilbert transform, given a Banach space $X$ and a CalderónZygmund operator $T$ with scalar-valued kernel $k$ one defines the vector-valued extension

$$
T_{X} f(x)=\sum_{k=1}^{m} T\left(\phi_{k}\right) x_{k} \in X
$$

for $f=\sum_{k=1}^{m} \phi_{k} x_{k}$ where $\phi_{k} \in L^{p}(\mathbb{R})$ and $x_{k} \in X$.

Next generalization in the theory comes from the fact that the expression $\int k(x, y) f(y) d y$ for vector valued functions $f$ also makes sense for operatorvalued kernels, with the obvious interpretation.

Let now $A$ and $B$ be Banach spaces and denote by $\mathcal{L}(A, B)$ the space of bounded linear operators from $A$ to $B$. Following Segovia and Torrea (see 
[ST1]) we shall say that $T$ is a $\mathcal{L}(A, B)$-Calderón-Zygmund type operator if it has following properties:

(i) $T: L^{p}\left(\mathbb{R}^{n}, A\right) \rightarrow L^{p}\left(\mathbb{R}^{n}, B\right)$ is bounded for some $1<p<\infty$ and

(ii) there exists a locally integrable function $k$ from $\mathbb{R}^{n} \times \mathbb{R}^{n} \backslash\{(x, x): x \in$ $\mathbb{R}^{n}$ \} into $\mathcal{L}(A, B)$ such that

$$
T f(x)=\int k(x, y) f(y) d y
$$

for every $A$-valued bounded and compactly supported function $f$ and $x \notin$ supp $f$.

Several properties can be imposed on these kernels in order to obtain the corresponding boundedness properties in different spaces:

$$
\begin{gathered}
\int_{|x-y| \geq 2\left|x-x^{\prime}\right|}\left\|k(x, y)-k\left(x^{\prime}, y\right)\right\| d y \leq C, \quad\left(H_{y}\right) \\
\int_{|x-y| \geq 2\left|y-y^{\prime}\right|}\left\|k(x, y)-k\left(x, y^{\prime}\right)\right\| d x \leq C, \quad\left(H_{x}\right) \\
\left\|k(x, y)-k\left(x^{\prime}, y\right)\right\| \leq C \frac{\left|x-x^{\prime}\right|^{\varepsilon}}{|x-y|^{n+\varepsilon}}, \quad|x-y| \geq 2\left|x-x^{\prime}\right|, \quad\left(C Z_{y}\right) \\
\left\|k(x, y)-k\left(x, y^{\prime}\right)\right\| \leq C \frac{\left|y-y^{\prime}\right|^{\varepsilon}}{|x-y|^{n+\varepsilon}}, \quad|x-y| \geq 2\left|y-y^{\prime}\right| . \quad\left(C Z_{x}\right)
\end{gathered}
$$

Note that $\left(C Z_{y}\right)$ (respect. $\left.\left(C Z_{x}\right)\right)$ implies $\left(H_{y}\right)$ (respect. $\left.\left(H_{x}\right)\right)$.

Throughout the paper we shall work on $\mathbb{R}^{n}$ endowed with the Lebesgue measure $d x$ and use the notation $|A|=\int_{A} d x$. Given a Banach space $(X, \|$. $\|)$ and $1 \leq p<\infty$ we shall denote by $L_{X}^{p}\left(\mathbb{R}^{n}\right)$ the space of Bochner $p$ integrable functions endowed with the norm $\|f\|_{L_{X}^{p}}=\left(\int_{\mathbb{R}^{n}}\|f(x)\|^{p} d x\right)^{1 / p}$ and by $L_{c}^{\infty}\left(\mathbb{R}^{n}, X\right)$ the closure of the compactly supported functions in $L_{X}^{\infty}\left(\mathbb{R}^{n}\right)$.

We use the notation $M f$ and $f^{\#}$ for the Hardy-Littlewood maximal function and the sharp maximal function of $f$, i.e.

$$
\begin{gathered}
M(f)(x)=\sup _{x \in Q} \frac{1}{|Q|} \int_{Q}\|f(x)\| d x, \\
\left.f^{\#}(x)=\sup _{x \in Q} \frac{1}{|Q|} \int_{Q}\left\|f(x)-\frac{1}{|Q|} \int_{Q} f(y) d y\right\| d x\right) .
\end{gathered}
$$

We shall denote $M_{q}(f)=M\left(\|f\|^{q}\right)^{1 / q}$ for $1<q<\infty$. We shall use the following results shown by Fefferman and Stein (see [FS, GR]):

$$
f^{\#}(x) \approx \sup _{x \in Q} \inf _{c_{Q} \in X} \frac{1}{|Q|} \int_{Q}\left\|f(x)-c_{Q}\right\| d x .
$$

If $M(f) \in L^{p_{0}}$ for some $0<p_{0}<\infty$ then for $1<p<\infty$ 


$$
\|f\|_{L_{X}^{p}\left(\mathbb{R}^{n}\right)} \leq C\left\|f^{\#}\right\|_{L^{p}\left(\mathbb{R}^{n}\right)} .
$$

We write $H_{X}^{1}\left(\mathbb{R}^{n}\right)$ for the Hardy space defined by $X$-valued atoms, consisting of integrable functions $f=\sum_{k} \lambda_{k} a_{k}$ where $\lambda_{k} \in \mathbb{R}, \sum_{k}\left|\lambda_{k}\right|<\infty$ and $a_{k}$ belong to $L_{c}^{\infty}\left(\mathbb{R}^{n}, X\right), \operatorname{supp}\left(a_{k}\right) \subset Q_{k}$ for some cube $Q_{k}, \int_{Q_{k}} a(x) d x=0$ and $\|a(x)\| \leq \frac{1}{\left|Q_{k}\right|}$.

Now $B M O_{X}\left(\mathbb{R}^{n}\right)$ stands for the space of locally integrable functions such that $\sup _{Q} \operatorname{osc}_{p}(f, Q)<\infty$ for some (or equivalently for all) $1 \leq p<\infty$, where

$$
\operatorname{osc}_{p}(f, Q)=\left(\frac{1}{|Q|} \int_{Q}\left\|f(x)-f_{Q}\right\|^{p} d x\right)^{1 / p}
$$

and $f_{Q}=\frac{1}{|Q|} \int_{Q} f(x) d x$ for a cube $Q$ in $\mathbb{R}^{n}$.

The interested reader should be aware that the duality $H^{1}(X)$ and $B M O\left(X^{*}\right)$ and the formulations of the spaces of $H_{X}^{1}$ or $B M O_{X}$ by means of the Hilbert transform are not longer true for infinite dimensional Banach spaces $X$. One needs either the RNP on $X^{*}$ or the $U M D$ property in $X$ (see [RRT, Bl1, Bl2]) for the corresponding results to hold true.

The following theorem exhibits the final achievements in the case $\mathcal{L}(A, B)$ valued kernels and goes back to the work of Benedek, Calderón and Panzone for convolution kernels and to the work of Rubio, Ruiz and Torrea for nonconvolution ones.

Theorem 1.2 ( $[B C P],[R R T])$ Let $A, B$ be Banach spaces and let $T$ be $\mathcal{L}(A, B)$-Calderón-Zygmund type operator satisfying $\left(H_{x}\right)$ and $\left(H_{y}\right)$. Then

(i) $T$ is weak-type $(1,1)$, i.e.

$$
\left|\left\{x:\|T f(x)\|_{B}>\lambda\right\}\right| \leq C \frac{\|f\|_{L_{A}^{1}}}{\lambda} .
$$

(ii) $T$ is bounded from $L_{A}^{q}\left(\mathbb{R}^{n}\right)$ to $L_{B}^{q}\left(\mathbb{R}^{n}\right)$.

Throughout the literature many results appeared in connection with the boundedness of commutators of Calderón-Zygmund type operators and multiplication by a function $b$ given by $T_{b}(f)=b T(f)-T(b f)$ on many different spaces, in the weighted and vector-valued settings. Let me now mention some result on operator-valued singular integrals which is one of the important contributions of Carlos Segovia to this theory.

Theorem 1.3 ([ST1, Theorem 1]) Let A, B be Banach spaces and let $T$ be an $\mathcal{L}(A, B)$-valued Calderón-Zygmund type operator such that the kernel satisfies $\left(C Z_{y}\right)$. Let $\ell \rightarrow \bar{\ell}$ be a correspondence from $\mathcal{L}(A)$ to $\mathcal{L}(B)$ such that

$$
\tilde{\ell} T(f)(x)=T(\ell f)(x)
$$

and

$$
k(x, y) \ell=\tilde{\ell} k(x, y) .
$$


If $b$ is $\mathcal{L}(A)$-valued, $b \in B M O_{\mathcal{L}(A)}\left(\mathbb{R}^{n}\right)$ and $\tilde{b} \in B M O_{\mathcal{L}(B)}\left(\mathbb{R}^{n}\right)$ then

$$
T_{b}(f)=b T(f)-T(b f)
$$

is bounded from $L_{A}^{p}\left(\mathbb{R}^{n}\right) \rightarrow L_{B}^{p}\left(\mathbb{R}^{n}\right)$ for all $1<p<\infty$.

Also endpoint estimates for the commutator was a topic that attracted several people on different directions (see [CP, HST, PP, P1, PT2]). The endpoint estimates of the previous operator-valued result were later studied by E. Harboure, C. Segovia and J.L. Torrea (see Theorem A and Theorem 3.1 in [HST]) when $b$ was assumed to be scalar-valued. We will not enter in this direction, but from their results one concludes that non-constant scalar valued $B M O$ functions do not define bounded commutators from $L_{c}^{\infty}\left(\mathbb{R}^{n}, A\right)$ to $B M O_{B}\left(\mathbb{R}^{n}\right)$ when kernel of the Calderón-Zygmund type operators are $\mathcal{L}(A, B)$-valued and also that, in general, $T_{b}$ does not map $H_{A}^{1}\left(\mathbb{R}^{n}\right)$ into $L_{B}^{1}\left(\mathbb{R}^{n}\right)$.

We shall present a proof of these last theorems in a bit more general situation and we recommend the interested reader to look for their applications to Maximal functions, Littlewood Paley theory and other topics in [RRT] and the work by Carlos in [ST1] (see also Chapter V in ([GR]) and ([ST2, ST3, ST4, ST5] for similar results in related operators and applications.)

As usual we denote $\lambda Q$ for a cube centered at $x_{Q}$ (center of $Q$ ) and with side length $\lambda \ell(Q)$, and $C$ will denote a constant that may vary from line to line.

\section{Theorems and proofs}

Although the vector-valued theory has been developed for operator-valued kernels, we shall see in what follows that most of the techniques used there can also be applied in a bit more general situation.

Definition 2.1 Let $A, B$ be Banach spaces and $1<p<\infty$. We say that $T$ is a $(p, A, B)$-Calderón Zygmund type operator if it is of weak-type $(p, p)$,

$$
\left|\left\{x:\|T f(x)\|_{B}>\lambda\right\}\right| \leq C \frac{\|f\|_{L_{A}^{p}}^{p}}{\lambda^{p}},
$$

and there exist a Banach space $E$, a bounded bilinear map $u: E \times A \rightarrow B$ and a locally integrable function $k$ from $\mathbb{R}^{n} \times \mathbb{R}^{n} \backslash\left\{(x, x): x \in \mathbb{R}^{n}\right\}$ into $E$ such that

$$
T f(x)=\int u(k(x, y), f(y)) d y
$$

for every A-valued simple function $f$ and $x \notin$ supp $f$.

We then say that $T$ has kernel $k$ with respect to $u$. 
We shall use the notation from the previous section $\left(H_{x}\right),\left(H_{y}\right),\left(C Z_{x}\right)$ and $\left(C Z_{y}\right)$ also when replacing the norm in $\mathcal{L}(A, B)$ by the norm in $E$.

As in the scalar case the basic ingredients in our proofs will be the Calderón-Zygmund decomposition and Kolmogorov inequality.

Lemma 2.2 ([GR], Theorem 1.2) Let $\phi$ be a non-negative integrable function and $\lambda>0$. There exists a sequence of disjoint dyadic cubes $\left\{Q_{j}\right\}$ such that

$$
\begin{gathered}
\phi(x) \leq \lambda, \text { a.a. } \notin \cup_{j} Q_{j}, \\
\left|\cup_{j} Q_{j}\right| \leq \frac{\|\phi\|_{1}}{\lambda}, \\
\lambda<\frac{1}{\left|Q_{j}\right|} \int_{Q_{j}} \phi \leq 2^{n} \lambda .
\end{gathered}
$$

We shall need the following Lemma due to Kolmogorov.

Lemma 2.3 Let $1 \leq q<p$ and $Q$ a cube. If $T$ is any operator of weak-type $(p, p)$ then

$$
\left(\frac{1}{|Q|} \int_{Q}\|T f(x)\|_{B}^{q} d x\right)^{1 / q} \leq C\left(\frac{1}{|Q|} \int_{Q}\|f(x)\|_{A}^{p} d x\right)^{1 / p} .
$$

Proof. Use the weak-type $(p, p)$ condition to estimate

$$
\begin{aligned}
\int_{Q}\|T f(x)\|^{q} d x & =q \int_{0}^{\infty} t^{q-1}|\{x \in Q:\|T f(x)\|>t\}| d t \\
& \leq q \int_{0}^{\infty} t^{q-1} \min \left\{|Q|, C^{p} \frac{\|f\|_{p}^{p}}{t^{p}}\right\} d t \\
& =q \int_{0}^{C \frac{\|f\|_{p}}{|Q|^{1 / p}}} t^{q-1}|Q| d t+q \int_{C \frac{\|f\|_{p}}{|Q|^{1 / p}}}^{\infty} C^{p} \frac{\|f\|_{p}^{p}}{t^{p-q+1}} d t \\
& \leq C|Q|^{1-q / p}\|f\|_{L_{A}^{p}}^{q} . \square
\end{aligned}
$$

Proposition 2.4 Let $A, B$ be Banach spaces. Let $T$ be a $(p, A, B)$-Calderón Zygmund type operator with kernel $k$ with respect to $u$ which satisfies $\left(H_{x}\right)$. Then

(i) $T$ maps $H_{A}^{1}\left(\mathbb{R}^{n}\right)$ to $L_{B}^{1}\left(\mathbb{R}^{n}\right)$.

(ii) $T$ is weak-type $(1,1)$.

Proof. (i) It suffices to show that $\|T(a)\|_{L_{B}^{1}} \leq C$ for any $A$-valued atom.

Let $a$ be an integrable function supported on a cube $Q$ and mean value zero with $\|a(x)\| \leq \frac{1}{|Q|}$. Using Lemma 2.3 for $q=1$,

$$
\int_{2 Q}\|T a(x)\| d x \leq C|Q|^{1-\frac{1}{p}}\|a\|_{L_{A}^{p}} \leq C|Q|\left(\frac{1}{|Q|} \int_{Q}\|a(x)\|^{p} d x\right)^{1 / p} \leq C .
$$


To analyze the other part we shall use $\left(H_{x}\right)$. For each $x \notin 2 Q$ then

$$
\begin{aligned}
& T a(x)=\int_{Q} u(k(x, y), a(y)) d y=\int_{Q} u\left(k(x, y)-k\left(x, x_{Q}\right), a(y)\right) d y . \\
& \int_{\mathbb{R}^{n} \backslash 2 Q}\|T a(x)\|_{B} d x \leq \int_{\mathbb{R}^{n} \backslash 2 Q} \int_{Q}\left\|u\left(k(x, y)-k\left(x, x_{Q}\right), a(y)\right)\right\| d y d x \\
& \leq \int_{Q}\left(\int_{|x-y|>2\left|y-x_{Q}\right|}\|u\|\left\|k(x, y)-k\left(x, x_{Q}\right)\right\| d x\right)\|a(y)\| d y \\
& \leq C\|a\|_{L_{A}^{1}} \leq C .
\end{aligned}
$$

(ii) Let $f \in L_{A}^{1}\left(\mathbb{R}^{n}\right) \cap L_{c}^{\infty}\left(\mathbb{R}^{n}, A\right)$ and $\lambda>0$. Now apply Lemma 2.2 to $\|f\|$ and denote $\Omega=\cup_{j} Q_{j}$. This allows us to decompose $f=g+b$ where

$$
g=\sum_{j}\left(\frac{1}{\left|Q_{j}\right|} \int_{Q_{j}} f\right) \chi_{Q_{j}}+f \chi_{\mathbb{R}^{n} \backslash \Omega}
$$

and

$$
b=\sum_{j}\left(f-\frac{1}{\left|Q_{j}\right|} \int_{Q_{j}} f\right) \chi_{Q_{j}}
$$

Observe that $\|g(x)\| \leq C \lambda$ a.e. and $\|g\|_{L_{A}^{1}} \leq\|f\|_{L_{A}^{1}}$. Therefore

$$
\begin{aligned}
\left|\left\{x:\|T f(x)\|_{B}>\lambda\right\}\right| & \leq\left|\left\{x:\|T g(x)\|_{B}>\lambda / 2\right\}\right|+\left|\left\{x:\|T b(x)\|_{B}>\lambda / 2\right\}\right| \\
& \leq \frac{C}{\lambda^{p}}\|T g\|_{L_{B}^{p}}^{p}+\left|\left\{x:\|T b(x)\|_{B}>\lambda / 2\right\}\right| \\
& \leq \frac{C}{\lambda^{p}}\|g\|_{L_{A}^{p}}^{p}+\left|\left\{x:\|T b(x)\|_{B}>\lambda / 2\right\}\right| \\
& \leq \frac{C}{\lambda} \int\|g(x)\|_{A} d x+\left|\left\{x:\|T b(x)\|_{B}>\lambda / 2\right\}\right| \\
& \leq \frac{C}{\lambda} \int\|f(x)\|_{A} d x+\left|\left\{x:\|T b(x)\|_{B}>\lambda / 2\right\}\right| .
\end{aligned}
$$

As above, if $a$ is an integrable function supported on a cube $Q$ and mean value zero then

$$
\int_{\mathbb{R}^{n} \backslash 2 Q}\|T a(x)\|_{B} d x \leq C\|a\|_{L_{A}^{1}} .
$$

In particular, denoting $b_{j}=\left(f-\frac{1}{\left|Q_{j}\right|} \int_{Q_{j}} f\right) \chi_{Q_{j}}$ one has

$$
\int_{\mathbb{R}^{n} \backslash 2 Q_{j}}\left\|T b_{j}(x)\right\| d x \leq C \int_{Q_{j}}\left\|b_{j}(y)\right\| d y \leq 2 C \int_{Q_{j}}\|f(y)\| d y .
$$

Using that $f \chi_{\Omega} \in L_{A}^{p}\left(\mathbb{R}^{n}\right)$ one easily gets that $\sum_{j} b_{j}$ converges in $L_{A}^{p}\left(\mathbb{R}^{n}\right)$. This implies that 


$$
\left\{x:\left\|T b(x)-\sum_{j=1}^{N} T b_{j}(x)\right\|>\varepsilon\right\} \mid \leq C \frac{\left\|b-\sum_{j=1}^{N} b_{j}\right\|_{L_{A}^{p}}^{p}}{\varepsilon^{p}}
$$

for any $\varepsilon>0$, which shows that $\|T b(x)\|_{B} \leq \sum_{j}\left\|T b_{j}(x)\right\|_{B}$ a.e.

This allows us to conclude that, for $\tilde{\Omega}=\cup_{j} 2 Q_{j}$,

$$
\begin{aligned}
\left|\left\{x:\|T b(x)\|_{B}>\lambda / 2\right\}\right| & \leq|\tilde{\Omega}|+\frac{2}{\lambda} \int_{\mathbb{R}^{n} \backslash \tilde{\Omega}}\|T b(x)\| d x \\
& \leq C|\Omega|+\frac{2}{\lambda} \sum_{j} \int_{\mathbb{R}^{n} \backslash 2 Q_{j}}\left\|T b_{j}(x)\right\| d x \\
& \leq C|\Omega|+\frac{2}{\lambda} \sum_{j} \int_{Q_{j}}\|f(x)\|_{A} d x \\
& \leq C \frac{\|f\|_{L_{A}^{1}}}{\lambda} .
\end{aligned}
$$

Proposition 2.5 Let $A, B$ be Banach spaces. Let $T$ be a $(p, A, B)$-Calderón Zygmund type operator with kernel $k$ with respect to $u$ which satisfies $\left(H_{y}\right)$. Then $T$ maps $L_{c}^{\infty}\left(\mathbb{R}^{n}, A\right)$ to $B M O_{B}\left(\mathbb{R}^{n}\right)$.

Proof. Let $f$ be $A$-valued simple function and let $Q$ be a cube in $\mathbb{R}^{n}$, decompose $f=f_{1}+f_{2}$ where $f_{1}=f \chi_{2 Q}$. Now write $c_{Q}=\left(T\left(f_{1}\right)\right)_{Q}-T\left(f_{2}\right)\left(x_{Q}\right)$ where $x_{Q}$ is the center of $Q$,

$$
T f(x)-c_{Q}=T\left(f_{1}\right)(x)-\left(T\left(f_{1}\right)\right)_{Q}+T\left(f_{2}\right)(x)-T\left(f_{2}\right)\left(x_{Q}\right) .
$$

Clearly, from Lemma 2.3,

$$
\begin{aligned}
\frac{1}{|Q|} \int_{Q}\left\|T\left(f_{1}\right)(x)-\left(T\left(f_{1}\right)\right)_{Q}\right\| d x & \leq \frac{2}{|Q|} \int_{Q}\left\|T\left(f_{1}\right)(x)\right\| d x \\
& \leq C\left(\frac{1}{|Q|} \int_{Q}\|f(x)\|^{p} d x\right)^{1 / p} \leq C\|f\|_{\infty} .
\end{aligned}
$$

On the other hand, for $x \in Q$ and $y \notin 2 Q$ one has $|x-y| \geq 2\left|x-x_{Q}\right|$, which implies

$$
T\left(f_{2}\right)(x)-T\left(f_{2}\right)\left(x_{Q}\right)=\int_{(2 Q)^{c}} u\left(k(x, y)-k\left(x_{Q}, y\right), f_{2}(y)\right) d y .
$$

Now the assumption $\left(H_{y}\right)$ gives

$$
\begin{aligned}
\left\|T\left(f_{2}\right)(x)-T\left(f_{2}\right)\left(x_{Q}\right)\right\| & \leq\|u\| \int_{(2 Q)^{c}}\left\|k(x, y)-k\left(x_{Q}, y\right)\right\|\|f(y)\| d y \\
& \leq C\|f\|_{\infty} \int_{|x-y| \geq 2\left|x-x_{Q}\right|}\left\|k(x, y)-k\left(x_{Q}, y\right)\right\| d y \\
& \leq C\|f\|_{\infty}
\end{aligned}
$$


Therefore $\frac{1}{|Q|} \int_{Q}\left\|T(f)(x)-c_{Q}\right\| d x \leq C\|f\|_{\infty}$. Which implies that $\|T f\|_{B M O} \leq$ $C\|f\|_{\infty}$ for any $f \in L_{c}^{\infty}\left(\mathbb{R}^{n}, A\right)$.

Using Propositions 2.4 and 2.5 together with interpolation one obtains the following result.

Theorem 2.6 Let $A, B$ be Banach spaces and let $T$ be a $(p, A, B)$-CalderónZygmund type operator and the kernel satisfies $\left(H_{x}\right)$ and $\left(H_{y}\right)$.

Then $T$ extends to a bounded operator from $L_{A}^{q}\left(\mathbb{R}^{n}\right)$ to $L_{B}^{q}\left(\mathbb{R}^{n}\right)$ for all $1<q<\infty$.

Proof. We can use the interpolation results (see $[\mathrm{Bl} 3, \mathrm{BX}]$ )

$$
\left[H_{A}^{1}\left(\mathbb{R}^{n}\right), L_{c}^{\infty}\left(\mathbb{R}^{n}, A\right)\right]_{\theta}=L_{A}^{p}\left(\mathbb{R}^{n}\right), \frac{1}{p}=1-\theta,
$$

and

$$
\left[L_{B}^{1}\left(\mathbb{R}^{n}\right), B M O_{B}\left(\mathbb{R}^{n}\right)\right]_{\theta}=L_{B}^{p}\left(\mathbb{R}^{n}\right), \frac{1}{p}=1-\theta . \square
$$

We shall now see that a condition slightly weaker than $\left(C Z_{y}\right)$ allows to get certain pointwise majoration which also gives the strong type in all $1<q<\infty$ for $(p, A, B)$-Calderón-Zygmund type operators.

Proposition 2.7 Let $A, B$ be Banach spaces and let $T$ be a $(p, A, B)$-CalderónZygmund type operator with kernel $k$ with respect to $u$. Assume the kernel satisfies that there exist $q>1$ and a sequence $\left(A_{j}\right)$ such that $\sum_{j=1}^{\infty} A_{j}<\infty$ where

$$
\sup _{\left|x-x^{\prime}\right| \leq R}\left(2^{j} R\right)^{n / q}\left(\int_{2^{j} R \leq|x-y|<2^{j+1} R}\left\|k(x, y)-k\left(x^{\prime}, y\right)\right\|^{q^{\prime}} d y\right)^{1 / q^{\prime}} \leq A_{j} .
$$

Then, for $s=\max \{p, q\}$,

$$
\left.T^{\#} f(x) \leq C M_{s}(f)(x)\right)
$$

for any A-valued bounded and compactly supported function $f$.

Proof. Let $x \in \mathbb{R}^{n}$ and let $Q$ be a cube centered at $x$ and radius $R$. Given a compactly supported and bounded function $f$, we write $f_{1}=f \chi_{2 Q}$ and $f_{2}=f-f_{1}$.

Note that (22) gives for any $z \in Q$

$$
T f_{2}(z)=\int_{|y-x|>2 R} u\left(k(z, y), f_{2}(y)\right) d y
$$

Denote $c_{Q}=T\left(f_{2}\right)(x)$ Therefore for $x^{\prime} \in Q$ 


$$
\begin{aligned}
\left\|T f_{2}\left(x^{\prime}\right)-c_{Q}\right\| & =\left\|\int_{|y-x|>2 R} u\left(k\left(x^{\prime}, y\right)-k(x, y), f_{2}(y)\right) d y\right\| \\
& =\left\|\int_{|y-x|>2 R} u\left(k\left(x^{\prime}, y\right)-k(x, y), f_{2}(y)\right) d y\right\| \\
& \leq\|u\| \int_{|y-x|>2 R}\left\|k\left(x^{\prime}, y\right)-k(x, y)\right\|\left\|f_{2}(y)\right\| d y \\
& \leq\|u\| \sum_{j=1}^{\infty} \int_{2^{j} R \leq|y-x|<2^{j+1} R}\left\|k\left(x^{\prime}, y\right)-k(x, y)\right\|\left\|f_{2}(y)\right\| d y \\
& \leq\|u\| \sum_{j=1}^{\infty}\left(2^{j} R\right)^{-n / q} A_{j}\left(\int_{2^{j}} R \leq|y-x|<2^{j+1} R\right. \\
& \left.\leq C\|u\| f_{2}(y) \|^{q} d y\right)^{1 / q} A_{j=1}\left(\frac{1}{2^{(j+1) n} R^{n}} \int_{|y-x|<2^{j+1} R}\|f(y)\|^{q} d y\right)^{1 / q} \\
& \leq C\|u\|\left(\sum_{j} A_{j}\right) M_{q} f(x)
\end{aligned}
$$

Hence

$$
\frac{1}{|Q|} \int_{Q}\left\|T f\left(x^{\prime}\right)-c_{Q}\right\| d x^{\prime} \leq \frac{1}{|Q|} \int_{Q}\left\|T f_{1}\left(x^{\prime}\right)\right\| d x^{\prime}+C\|u\| M_{q} f(x) .
$$

Using Lemma 2.3 one gets

$$
\frac{1}{|Q|} \int_{Q}\left\|T f_{1}\left(x^{\prime}\right)\right\| d x^{\prime} \leq C\left(\frac{1}{|Q|} \int_{2 Q}\|f(y)\|^{p} d y\right)^{1 / p} \leq C M_{p}(f)(x) .
$$

Therefore $(T f)^{\#}(x) \leq C\left(M_{p}(f)(x)+M_{q}(f)(x)\right) \leq C M_{s}(f)(x)$.

\section{Commutators}

Let us define a notion which is needed for our purposes.

Definition 3.1 Let $T$ be a bounded operator from $L_{A}^{p}\left(\mathbb{R}^{n}\right)$ to $L_{B}^{p}\left(\mathbb{R}^{n}\right)$, and let $b_{1}$ and $b_{2}$ be $\mathcal{L}(A)$ and $\mathcal{L}(B)$ - valued functions respectively. Define

$$
T_{b_{1}, b_{2}}(f)=b_{2} T(f)-T\left(b_{1} f\right)
$$

for any A-valued simple function $f$.

We shall be using the following basic assumptions for our general version of the commutator theorem. 
Definition 3.2 Let $T$ be a $(p, A, B)$-Calderón-Zygmund type operator with kernel $k$ with respect to $u$ and let $b_{1}$ and $b_{2}$ be $\mathcal{L}(A)$ and $\mathcal{L}(B)$-valued functions respectively. We shall say that $\left(b_{1}, b_{2}\right)$ has the commuting properties $(C P)_{1}$ and $(C P)_{2}$ if

$(C P)_{1} \quad b_{2}(z) u(k(x, y), a)=u\left(k(x, y), b_{1}(z) a\right), \quad x, y, z \in \mathbb{R}^{n}, x \neq y$.

$(C P)_{2} \quad\left(b_{2}\right)_{Q} T\left(a \chi_{A}\right)\left(x^{\prime}\right)=T\left(\left(b_{1}\right)_{Q} a \chi_{A}\right)\left(x^{\prime}\right)$ for any $Q$ cube, $A \subset Q$, $a \in A$ and $x^{\prime} \in Q$.

We would like to point out that $(C P)_{1}$ produces the following cancelation property.

Lemma 3.3 Let $\left(b_{1}, b_{2}\right)$ satisfy $(C P)_{1}$, let $Q, Q^{\prime}$ be cubes in $\mathbb{R}^{n}$ and $f_{1}$ and $f_{2}$ be simple A-valued with supp $f_{1} \subset Q^{\prime}$ and supp $f_{2} \subset\left(Q^{\prime}\right)^{c}$. Then

$$
\begin{gathered}
\left(b_{2}\right)_{Q} T\left(f_{1}\right)(x)=T\left(\left(b_{1}\right)_{Q} f_{1}\right)(x), \quad x \in\left(Q^{\prime}\right)^{c} \\
\left(b_{2}\right)_{Q} T\left(f_{2}\right)(x)=T\left(\left(b_{1}\right)_{Q} f_{2}\right)(x), \quad x \in Q^{\prime} .
\end{gathered}
$$

Proof. We prove only (28), the other case follows in a similar way.

Recall that if $F \in L^{1}\left(\mathbb{R}^{n}, X\right)$ and $\Phi \in \mathcal{L}(X)$ for a given Banach space then $\Phi\left(\int F(x) d x\right)=\int \Phi F(x) d x$. Hence

$$
\begin{aligned}
\left(b_{2}\right)_{Q} T\left(f_{2}\right)(x) & =\left(b_{2}\right)_{Q}\left(\int_{\left(Q^{\prime}\right)^{c}} u\left(k(x, y), f_{2}(y)\right) d y\right. \\
& =\int_{\left(Q^{\prime}\right)^{c}}\left(b_{2}\right)_{Q} u\left(k(x, y), f_{2}(y)\right) d y \\
& =\int_{\left(Q^{\prime}\right)^{c}}\left(\frac{1}{|Q|} \int_{Q} b_{2}(z) d z\right) u\left(k(x, y), f_{2}(y)\right) d y \\
& =\int_{\left(Q^{\prime}\right)^{c}}\left(\frac{1}{|Q|} \int_{Q} b_{2}(z) u\left(k(x, y), f_{2}(y) d z\right) d y\right. \\
& =\int_{\left(Q^{\prime}\right)^{c}}\left(\frac{1}{|Q|} \int_{Q} u\left(k(x, y), b_{1}(z) f_{2}(y)\right) d z\right) d y \\
& =\int_{\left(Q^{\prime}\right)^{c}} u\left(k(x, y),\left(\frac{1}{|Q|} \int_{Q} b_{1}(z) d z\right) f_{2}(y)\right) d y \\
& =T\left(\left(b_{1}\right)_{Q} f_{2}\right)(x) . \square
\end{aligned}
$$

Example 3.4 Let $S_{1}: A \rightarrow B, S_{2}: B \rightarrow B$ and $S: A \rightarrow B$ be bounded operators such that $S_{2} S=S S_{1}$. Let $u: \mathbb{C} \times A \rightarrow B$ be given by $(\lambda, a) \rightarrow \lambda S a$ and $k$ be a scalar-valued kernel. Define $T_{S}\left(\sum_{j=1}^{N} \phi_{k} a_{k}\right)=\sum_{j=1}^{N} T\left(\phi_{k}\right) S\left(a_{k}\right)$ where $T$ is the scalar-valued Calderón-Zygmund operator with kernel $k$.

If $b_{1}(x)=b(x) S_{1}$ and $b_{2}(x)=b(x) S_{2}$ for some scalar valued functions $b(x)$ then $\left(b_{1}, b_{2}\right)$ has the commuting properties $(C P)_{1}$ and $(C P)_{2}$. In this case one has

$$
T_{b_{1}, b_{2}}(f)(x)=b(x) S_{2}\left(T_{S} f(x)\right)-T_{S}\left(b S_{1} f\right)(x) .
$$


Example 3.5 Let $A$ be a Banach space and let $u: A^{*} \times A \rightarrow \mathbb{C}$ be given by $u\left(a^{*}, a\right)=\left\langle a^{*}, a\right\rangle$. Let $k$ be $A^{*}$-valued function and let $T$ be a CalderónZygmund operator from $L_{A}^{p}(\mathbb{R})$ into $L^{p}(\mathbb{R})$ with kernel $k$ with respect to $u$.

If $b_{1}(x)=b(x) I d_{A}$ and $b_{2}(x)=b(x)$ for a scalar valued $b$ then $\left(b_{1}, b_{2}\right)$ has the commuting properties $(C P)_{1}$ and $(C P)_{2}$. In this case one has

$$
T_{b_{1}, b_{2}}(f)(x)=b(x) T f(x)-T(b f)(x) .
$$

Example 3.6 Let $b(x) \in A^{*}$ and let $u: \mathbb{C} \times A \rightarrow A$ given by $(\lambda, a) \rightarrow \lambda a$. Let $k(x, y)$ be a scalar valued function and $T$ a Calderón-Zygmund operator with kernel $k$ and denote by $T_{A}=T \otimes I d_{A}$. If $b_{1}(x)(a)=b_{2}(x)(a)=\langle b(x), a\rangle a_{0}$ for a fixed $a_{0} \in A$ then $\left(b_{1}, b_{2}\right)$ has the commuting properties $(C P)_{1}$ and $(C P)_{2}$. In this case one has

$$
T_{b_{1}, b_{2}}(f)=\left(\left\langle b, T_{A}(f)\right\rangle-T(\langle b, f\rangle) a_{0} .\right.
$$

Let us start with the basic facts which follow from $(C P)_{1}$ and $(C P)_{2}$.

Lemma 3.7 Let $\left(b_{1}, b_{2}\right)$ satisfy $(C P)_{1}$ and $(C P)_{2}$, let $Q$ be cube in $\mathbb{R}^{n}$ and $f$ be simple A-valued. Then

$$
\left(b_{2}\right)_{Q} T(f)(x)=T\left(\left(b_{1}\right)_{Q} f\right)(x) x \in Q .
$$

Proof. Take $f_{1}=f \chi_{Q}$ and $f_{2}=f-f_{1}$. and invoke Lemma 3.3 to obtain $\left(b_{2}\right)_{Q} T\left(f_{2}\right) \chi_{Q}=T\left(\left(b_{1}\right)_{Q} f_{2}\right) \chi_{Q}$. Now $(C P)_{2}$ gives that $\left(b_{2}\right)_{Q} T\left(f_{1}\right) \chi_{Q}=$ $T\left(\left(b_{1}\right)_{Q} f_{1}\right) \chi_{Q}$.

Another useful lemma which is essentially included in [HST] is the following

Lemma 3.8 Let $T$ be $(p, A, B)$-Calderón-Zygmund type operator with kernel $k$ with respect to $u$ and which satisfes the assumption $\left(C Z_{y}\right)$. If $f$ is compactly supported $A$-valued with supp $f \subset(2 Q)^{c}$ then

$$
\left\|T(f)(x)-T(f)\left(x^{\prime}\right)\right\| \leq C\|u\| \frac{\left|x-x^{\prime}\right|^{\varepsilon}}{\ell(Q)^{\varepsilon}} \sum_{j=2}^{\infty} \frac{2^{-j \varepsilon}}{\left|Q_{j}\right|} \int_{Q_{j}}\|f(y)\| d y, \quad x, x^{\prime} \in Q
$$

where we are denoting $Q_{j}=2^{j} Q$

Proof. Note that if $x, x^{\prime} \in Q$ then

$$
T(f)(x)-T(f)\left(x^{\prime}\right)=\int_{(2 Q)^{c}} u\left(k(x, y)-k\left(x^{\prime}, y\right), f(y)\right) d y .
$$




$$
\begin{aligned}
\left\|T(f)(x)-T(f)\left(x^{\prime}\right)\right\| & \leq\|u\| \int_{(2 Q)^{c}}\left\|k(x, y)-k\left(x^{\prime}, y\right)\right\|\|f(y)\| d y \\
& \leq C\|u\|\left|x-x^{\prime}\right|^{\varepsilon} \int_{(2 Q)^{c}} \frac{\|f(y)\|}{|x-y|^{n+\varepsilon}} d y \\
& \leq C\|u\|\left|x-x^{\prime}\right|^{\varepsilon} \sum_{j=1}^{\infty} \int_{Q_{j+1}-Q_{j}} \frac{\|f(y)\|}{|x-y|^{n+\varepsilon}} d y \\
& \leq C\|u\|\left|x-x^{\prime}\right|^{\varepsilon} \sum_{j=2}^{\infty} \frac{1}{\ell\left(Q_{j}\right)^{n+\varepsilon}} \int_{Q_{j}}\|f(y)\| d y \\
& \leq C\|u\| \frac{\left|x-x^{\prime}\right|^{\varepsilon}}{\ell(Q)^{\varepsilon}} \sum_{j=2}^{\infty} 2^{-j \varepsilon} \frac{1}{\left|Q_{j}\right|} \int_{Q_{j}}\|f(y)\| d y . \square
\end{aligned}
$$

We shall present now our result on commutators. It is just an adaptation of the proof in [ST1] under slightly weaker assumptions.

Proposition 3.9 Let $b_{1} \in B M O\left(\mathbb{R}^{n}, \mathcal{L}(A)\right), b_{2} \in B M O\left(\mathbb{R}^{n}, \mathcal{L}(B)\right)$ and $1<p<\infty$. Let $T$ be a $(p, A, B)$ - Calderón-Zygmund type operator with kernel $k$ with respect to $u$ which satisfies $\left(C Z_{y}\right)$. Let $\left(b_{1}, b_{2}\right)$ satisfy $(C P)_{1}$ and $(\mathrm{CP})_{2}$. Then, for any $1<q<p<s<\infty$, there exists $C_{q, s}>0$ such that

$$
T_{b_{1}, b_{2}}(f)^{\#}(x) \leq C_{q, s}\left(\left\|b_{2}\right\|_{B M O} M_{q}(T f)(x)+\left\|b_{1}\right\|_{B M O} M_{s}(f)(x)\right) .
$$

Proof. Let $f$ be a simple $E$-valued function. Let $Q$ be a cube and denote $f_{1}=f \chi_{2 Q}$ and $f_{2}=f-f_{1}$. Put $c_{Q}=T\left(\left[\left(b_{1}\right)_{Q}-b_{1}\right] f_{2}\right)\left(x_{Q}\right)$.

For each $x \in Q$ one has, applying Lemma 3.7,

$$
\begin{gathered}
T_{b_{1}, b_{2}} f(x)=b_{2} T f(x)-T\left(b_{1} f\right)(x)= \\
=\left[b_{2}-\left(b_{2}\right)_{Q}\right] T f(x)+T\left(\left[\left(b_{1}\right)_{Q}-b_{1}\right] f\right)(x)= \\
=\left[b_{2}-\left(b_{2}\right)_{Q}\right] T f(x)+T\left(\left[\left(b_{1}\right)_{Q}-b_{1}\right] f_{1}\right)(x)+T\left(\left[\left(b_{1}\right)_{Q}-b_{1}\right] f_{2}\right)(x) .
\end{gathered}
$$

Hence

$$
T_{b_{1}, b_{2}} f(x)-c_{Q}=\sum_{i=1}^{3} \sigma_{i}(x)
$$

where

$$
\begin{gathered}
\sigma_{1}(x)=\left[b_{2}-\left(b_{2}\right)_{Q}\right] T f(x), \\
\sigma_{2}(x)=T\left(\left[\left(b_{1}\right)_{Q}-b_{1}\right] f_{1}\right)(x)
\end{gathered}
$$

and

$$
\sigma_{3}(x)=T\left(\left[\left(b_{1}\right)_{Q}-b_{1}\right] f_{2}\right)(x)-T\left(\left[\left(b_{1}\right)_{Q}-b_{1}\right] f_{2}\right)\left(x_{Q}\right) .
$$

Observe that for any $q>1$ and $1 / q+1 / q^{\prime}=1$ we can write

$$
\frac{1}{|Q|} \int_{Q}\left\|\sigma_{1}(x)\right\| d x \leq \operatorname{osc}_{q^{\prime}}\left(b_{2}, Q\right)\left(\frac{1}{|Q|} \int_{Q}\|T f(x)\|^{q} d x\right)^{1 / q} .
$$


For any $s>p>q_{1}>1$ one can write, for $1 / s+1 / r=1 / p$, from Lemma 2.3 ,

$$
\begin{aligned}
\frac{1}{|Q|} \int_{Q}\left\|\sigma_{2}(x)\right\| d x & \leq\left(\frac{1}{|Q|} \int_{Q}\left\|T\left(\left[\left(b_{1}\right)_{Q}-b_{1}\right] f_{1}\right)(x)\right\|^{q_{1}} d x\right)^{1 / q_{1}} \\
& \leq C\left(\frac{1}{|Q|} \int_{Q}\left\|\left(b_{1}-\left(b_{1}\right)_{Q}\right) f_{1}(x)\right\|^{p} d x\right)^{1 / p} \\
& \leq \operatorname{Cosc}_{r}\left(b_{1}, Q\right)\left(\frac{1}{|Q|} \int_{Q}\|f(x)\|^{s} d x\right)^{1 / s} .
\end{aligned}
$$

Using Lemma 3.8, and taking into account that $\left\|\left(b_{1}\right)_{Q}-\left(b_{1}\right)_{2 Q}\right\| \leq$ $\operatorname{Cosc}_{q_{1}}\left(b_{1}, 2 Q\right)$, we also can estimate, for a given $s>1$,

$$
\begin{aligned}
\left\|\sigma_{3}(x)\right\| & \leq C \sum_{j=2}^{\infty} 2^{-j \varepsilon} \frac{1}{\left|Q_{j}\right|} \int_{Q_{j}}\left\|\left(b_{1}(y)-\left(b_{1}\right)_{Q}\right) f(y)\right\| d y \\
& \leq C \sum_{j=2}^{\infty} 2^{-j \varepsilon}\left(\frac{1}{\left|Q_{j}\right|} \int_{Q_{j}}\left\|b_{1}(y)-\left(b_{1}\right)_{Q}\right\|^{s^{\prime}} d y\right)^{1 / s^{\prime}}\left(\frac{1}{\left|Q_{j}\right|} \int_{Q_{j}}\|f(y)\|^{s} d y\right)^{1 / s} \\
& \leq C \sum_{j=2}^{\infty} 2^{-j \varepsilon}\left(\sum_{k=2}^{j} \operatorname{osc}_{s^{\prime}}\left(b_{1}, Q_{k}\right)\right)\left(\frac{1}{\left|Q_{j}\right|} \int_{Q_{j}}\|f(y)\|^{s} d y\right)^{1 / s} \\
& \left.\leq C \sup _{j \geq 2} \frac{1}{\left|Q_{j}\right|} \int_{Q_{j}}\|f(y)\|^{s} d y\right)^{1 / s}\left(\sum_{j=2}^{\infty} 2^{-j \varepsilon}\left(\sum_{k=2}^{j} o s c_{q^{\prime}}\left(b_{1}, Q_{k}\right)\right)\right) \\
& \leq C\left\|b_{1}\right\|_{B M O} \sup _{j \geq 2}\left(\frac{1}{\left|Q_{j}\right|} \int_{Q_{j}}\|f(y)\|^{s} d y\right)^{1 / s} \sum_{j} j 2^{-j \varepsilon} \\
& \leq C\left\|b_{1}\right\|_{B M O} M_{s}(f)(x) .
\end{aligned}
$$

Hence, combining the previous estimates, one obtains

$$
\left.T_{b_{1}, b_{2}}(f)^{\#}(x) \leq C\left\|b_{2}\right\|_{B M O} M_{q}(T f)(x)+C\left\|b_{1}\right\|_{B M O} M_{s}(f)(x)\right) . \square
$$

Theorem 3.10 Let $b_{1} \in B M O\left(\mathbb{R}^{n}, \mathcal{L}(A)\right)$ and $b_{2} \in B M O\left(\mathbb{R}^{n}, \mathcal{L}(B)\right)$. Let $T$ be a $(p, A, B)$ - Calderón-Zygmund type operator with kernel $k$ with respect to $u$ which satisfy $\left(H_{x}\right)$ and $\left(C Z_{y}\right)$ and let $\left(b_{1}, b_{2}\right)$ satisfy $(C P)_{1}$ and $(C P)_{2}$. Then $T_{b_{1}, b_{2}}$ is bounded from $L_{A}^{q}\left(\mathbb{R}^{n}\right)$ to $L_{B}^{q}\left(\mathbb{R}^{n}\right)$ for any $1<q<\infty$.

Proof. First use Theorem 2.6 to obtain that $T$ is a $(s, A, B)$ - CalderónZygmund type operator for all $1<s<p$. In fact $T$ is bounded from $L_{A}^{r}\left(\mathbb{R}^{n}\right)$ to $L_{A}^{r}\left(\mathbb{R}^{n}\right)$ for all $1<r<\infty$. Use, for a given $1<q<\infty$, Proposition 3.9 for $1<q_{1}, s_{1}<q$, which, combining the boundedness of $M_{q_{1}}$ and $M_{s_{1}}$ in $L_{B}^{q}\left(\mathbb{R}^{n}\right)$ and the boundedness of $T$ from $L_{A}^{q}\left(\mathbb{R}^{n}\right)$ to $L_{B}^{q}\left(\mathbb{R}^{n}\right)$, imply that

$$
\begin{aligned}
\left\|T_{b_{1}, b_{2}} f\right\|_{L_{B}^{q}\left(\mathbb{R}^{n}\right)} & \leq C\left\|T_{b_{1}, b_{2}}(f)^{\#}\right\|_{L_{B}^{q}\left(\mathbb{R}^{n}\right)} \\
& \leq C\left(\left\|b_{2}\right\|_{B M O}\left\|M_{q_{1}}(T f)\right\|_{L^{q}}+C\left\|b_{1}\right\|_{B M O}\left\|M_{s_{1}}(f)\right\|_{L^{q}}\right) \\
& \leq C\left(\left\|b_{2}\right\|_{B M O}\|T\|\|f\|_{L^{q}}+C\left\|b_{1}\right\|_{B M O}\|f\|_{L^{q}}\right) . \square
\end{aligned}
$$




\section{References}

[BCP] Benedek, A.; Calderón, A.P.; Panzone, R. Convolution operators on Banach space valued functions, Proc. Nat. Acad. Sci. U.S.A., 48, (1962), 356-365.

[Bl1] Blasco, O. Hardy spaces of vector-valued functions:Duality, Trans. Amer. Math. Soc. 308(1988), 495-507.

[Bl2] Blasco, O. Boundary values of functions in vector-valued Hardy spaces and geometry of Banach spaces, J. Funct. Anal. 78(1988), 346-364.

[Bl3] Blasco, O. Interpolation between $H_{B_{0}}^{1}$ and $L_{B_{1}}^{p}$. Studia Math. 92(1989), no. 3, 205-210.

[BX] Blasco, O.; Xu, Q. Interpolation between vector-valued Hardy spaces. J. Funct. Anal. 102 (1991), no. 2, 331-359.

[B] Bloom, S. A commutator theorem and weighted BMO, Trans. Amer. Math. Soc. 292, (1985), 103-122.

[Bo1] Bourgain, J. Some remarks on Banach spaces in which martingale differences are unconditional, Ark. Mat 21, (1983), 163-168.

[Bo2] Bourgain, J. Extension of a result of Benedek, Calderón and Panzone, Ark. Mat 22, (1984), 91-95.

[Bu1] Burkholder, D.L. A geometrical characterization of the Banach spaces in which martingale differences are unconditional, Ann. of Prob, 9, (1981), 9971011.

[Bu2] Burkholder, D.L. A geometric condition that implies the existence of certain singular integrals of Banach-space-valued functions, Conf. Harmonic Analysis in honor of A. Zygmund, (W. Beckner, A.P. Calderón, R. Fefferman and P.W. Jones editors). Wadsworth Inc., 1981, 270-286.

[CZ] Calderón, A; Zygmund, A. On the existence of certain singular integrals. Acta Math. 88 (1952), 85-139.

[C] Coifman, A real variable characterization of $H^{p}$. Studia Math. 51, (1974), 269274.

[CM] Coifman, R.; Meyer, Y. Au-dèlá des opérateurs pseudodiffer'entiels. Astérisque 57, Soc. Math. France, Paris, 1978.

[CRW] Coifman, R.; Rochberg, R.; Weiss, G. Factorization theorems for Hardy spaces in several variables. Ann. of Math. 103 (1976), no. 2, 611-635.

[CP] Cruz-Uribe, D.; Pérez, C. Two-weight, weak-type norm inequalities for fractional integrals, Calderón-Zygmund operators and commutators. Indiana Univ. Math. J. 49 (2000), no. 2, 697-721.

[D] Duoandikoetxea, J. Fourier Analysis. Graduate Studies in Mathematics, 29 American Mathematical Society, Providence, RI, 2001.

[FS] C. Fefferman, E. M. Stein, $H^{p}$ spaces of several variables, Acta Math. 129 (1972), 137-193.

[GMT] García-Cuerva, J.; Macías, R.; Torrea, J. L. The Hardy-Littlewood property of Banach lattices. Israel J. Math. 83 (1993), no. 1-2, 177-201.

[GMT2] García-Cuerva, J.; Macías, R. A.; Torrea, J. L. Maximal operators and B.M.O. for Banach lattices. Proc. Edinburgh Math. Soc. (2) 41 (1998), no. 3, 585-609.

[GR] García-Cuerva, J.; Rubio de Francia, J.L. Weighted norm inequalities and related topics, North-Holland, Amsterdam, 1985.

[HMST] Harboure, E.; Macías, R. A.; Segovia, C.; Torrea, J. L. Some estimates for maximal functions on Köthe function spaces. Israel J. Math. 90 (1995), no. 1-3, $349-371$. 
[HST] Harboure, E., Segovia, C., Torrea, J.L. Boundedness of commutators of fractional and singular integrals for the extreme values of $p$, Illinois J. Math. 41, no. 4 (1997), 676-700.

[H] Hörmander, L. Estimates for translation invariant operators in $L^{p}$ spaces, Acta Math. 104, (1960), 93-140.

[J] Journé, J.L. Calderón-Zygmund Operators, Pseudo-differential Operators and the Cauchy integral of Calderón, Lecture Notes 994, Springer Verlag, Berlin, 1983

[P1] Pérez, C. Endpoint estimates for commutators of singular integral operators, J. Funct. Anal. 128, (1995), 163-185.

[PP] Pérez, C.; Pradolini, G.Sharp weighted endpoint estimates for commutators of singular integrals. Michigan Math. J. 49 (2001), no. 1, 23-37.

[PT2] Pérez, C.; Trujillo-González, R. Sharp weighted estimates for vector-valued singular integral operators and commutators. Tohoku Math. J. (2) 55 (2003), no. 1, 109-129.

[RRT] Rubio de Francia,J.L.; Ruiz,F.; Torrea,J.L. Calderón-Zygmund theory for vector-valued functions, Adv. in Math. 62 (1986), 7-48.

[ST1] Segovia, C.; Torrea, J.L. Vector-valued commutators and applications, Indiana Univ. Math. J. 38 (1989), no. 4, 959-971.

[ST2] Segovia, C,; Torrea, J. L. A note on the commutator of the Hilbert transform. Rev. Un. Mat. Argentina 35 (1989), 259-264.

[ST3] Segovia, C.; Torrea, J. L. Weighted inequalities for commutators of fractional and singular integrals. Conference on Mathematical Analysis (El Escorial, 1989). Publ. Mat. 35 (1991), no. 1, 209-235.

[ST4] Segovia, C.; Torrea, J. L. Commutators of Littlewood-Paley sums. Ark. Mat. 31 (1993), no. 1, 117-136.

[ST5] Segovia, C.; Torrea, J. L. Higher order commutators for vector-valued Calderón-Zygmund operators. Trans. Amer. Math. Soc. 336 (1993), no. 2, $537-$ 556.

[S] Stein, E. M. Singular integrals and differentiability properties of functions , Princeton Univ. Press, [1970].

[SW] E. M. Stein and G. Weiss, Introduction to Fourier Analysis on Euclidean Spaces, Princeton Univ. Press, [1971]. 